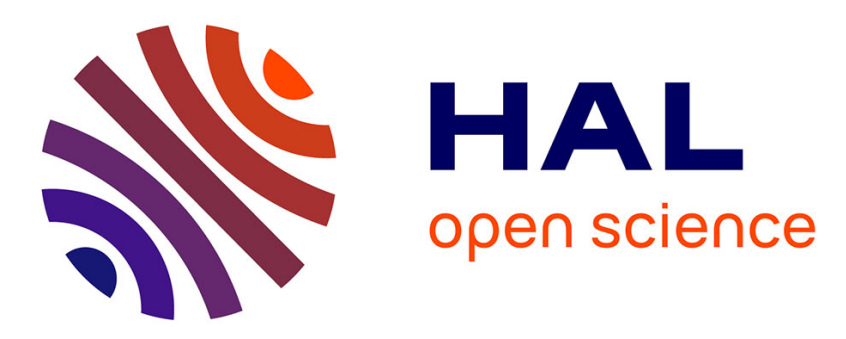

\title{
Issues and challenges for pedestrian active safety systems based on real world accidents
}

Hedi Hamdane, Thierry Serre, Catherine Masson, Robert Anderson

\section{To cite this version:}

Hedi Hamdane, Thierry Serre, Catherine Masson, Robert Anderson. Issues and challenges for pedestrian active safety systems based on real world accidents. Accident Analysis \& Prevention, 2015, 82, pp. 53-60. 10.1016/j.aap.2015.05.014. hal-01197212v3

\section{HAL Id: hal-01197212 \\ https://hal.science/hal-01197212v3}

Submitted on 19 Oct 2015

HAL is a multi-disciplinary open access archive for the deposit and dissemination of scientific research documents, whether they are published or not. The documents may come from teaching and research institutions in France or abroad, or from public or private research centers.
L'archive ouverte pluridisciplinaire HAL, est destinée au dépôt et à la diffusion de documents scientifiques de niveau recherche, publiés ou non, émanant des établissements d'enseignement et de recherche français ou étrangers, des laboratoires publics ou privés. 
Issues and challenges for pedestrian active safety systems based on real world accidents

Hédi HAMDANE

IFSTTAR-LMA

Aix-Marseille University, IFSTTAR-LBA

The University of Adelaide, CASR

hedi.hamdane@ifsttar.fr

Thierry SERRE*

IFSTTAR-LMA

Chemin de la Croix Blanche, 13300 Salon de Provence, France

thierry.serre@ifsttar.fr

(tel: +334905686 53; fax: +334905686 18)

\author{
Catherine MASSON \\ IFSTTAR-LBA
}

Faculté de médecine secteur Nord, F-13916 Marseille Cedex 20, France

catherine.masson@ifsttar.fr

\section{Robert ANDERSON}

Centre for Automotive Safety Research - CASR

The University of Adelaide, SA 5005, Australia

robert@casr.adelaide.edu.au

* Corresponding author: 
Issues and challenges for pedestrian active safety systems

based on real world accidents

\begin{abstract}
The purpose of this study was to analyze real crashes involving pedestrians in order to evaluate the potential effectiveness of Autonomous Emergency Braking systems (AEB) in pedestrian protection. A sample of 100 real accident cases were reconstructed providing a comprehensive set of data describing the interaction between the vehicle, the environment and the pedestrian all along the scenario of the accident. A generic AEB system based on a camera sensor for pedestrian detection was modeled in order to identify the functionality of its different attributes in the timeline of each crash scenario. These attributes were assessed to determine their impact on pedestrian safety. The influence of the detection and the activation of the AEB system were explored by varying the Field Of View (FOV) of the sensor and the level of deceleration. A FOV of $35^{\circ}$ was estimated to be required to detect and react to the majority of crash scenarios. For the reaction of a system (from hazard detection to triggering the brakes), between 0,5 and $1 \mathrm{~s}$ appears necessary.
\end{abstract}

Keywords: AEB systems, Pedestrian detection, Safety performance, Accident reconstruction

\title{
1. Introduction
}

Every year, more than 1.2 million people in the world die in traffic accidents and among these casualties $22 \%$ are pedestrians (WHO, 2013). This proportion of fatalities that are pedestrians is $14 \%$ in Australia and 16\% in the EU-15 (OECD/ITF, 2013). Worldwide organizations such as the OECD (Organization for Economic Co-operation and Development) and the WHO (World Health Organization) have outlined a set of objectives and actions to enhance pedestrian safety. Among these measures, the development of new safety-based technologies in the vehicle is promoted. Passive safety measures such as energy absorbing bumpers and hoods are designed to reduce the injury outcomes for pedestrians. These devices are evaluated under regulatory and non-regulatory tests (EuroNCAP). Along with these passive systems, active safety systems are being developed and introduced to prevent crashes. These active systems use sensors to monitor the forward path of the vehicle in order to detect a pedestrian. Once a hazard is detected, these systems trigger various countermeasures to avoid or mitigate collisions. These measures may include autonomous emergency braking or autonomous steering (e.g. Broggi et al., 2009; Hayashi et al., 2012; Keller et al., 2011a).

Active safety systems are mainly composed of the three following components: sensors for detection, a unit for processing and actuators for triggering an emergency maneuver. Concerning the first component, in order to detect various obstacles, cameras operating in visible light or infrared radiation (Near, Mid, Far) as well as RADARs and Laser Scanners are used. These different sensors have complementary functions as described by Gandhi and Trivedi (2007). So the combination of multiple sensors allows mare accurate detection. This is achieved by merging and filtering the data collected from the environment in order to distinguish pedestrians from other background obstacles. As soon as pedestrians are detected, 
they are tracked in order to predict any collision. If a crash is imminent, the system applies the appropriate countermeasure.

Several methods have been developed to assess the effectiveness of these systems and estimate their safety impact on real world crashes. Approaches based on numerical simulation have been explored to assess the effect of systems in various accident scenarios. These scenarios are provided from in-depth crash investigations and are simulated using simple models (Rosén et al., 2010; Seiniger et al., 2013). More complex methods (probabilistic methods as Monte Carlo) expand the scenarios by slightly varying the initial pre-crash conditions in order to cover a wider range of crash configurations (Lindman and Tivesten, 2006). Besides computational simulations, researchers are currently attempting to develop standardized on-track tests to assess the performance of these systems. However, these track tests are limited in numbers of test scenarios even if they are focusing on reference scenarios defined through cluster and statistical analyses of real world crashes (Ebner et al., 2011; Eckert et al., 2013; Lenard et al., 2014; Wisch et al., 2013).

Several active systems for pedestrian safety have already been introduced on the market such as the CWAB-PD (Collision Warning with Full Auto Brake and Pedestrian Detection) firstly used in the Volvo S60 MY2011 and the Eyesight system available in Subaru's 2013 models. The regulations and protocols are being developed (Schram et al., 2013) and some studies on the effectiveness of these active systems have been already presented. For example, Lindman et al. (2010) used a simulation based method to conclude that the CWAB-PD system may lead to a reduction of about $30 \%$ of all front-end accidents and $24 \%$ of pedestrian fatalities. Ando and Tanaka (2013) assessed two systems using "standard tests" (vehicle driving towards a pedestrian dummy positioned on the course). The results attest that the functionality of the systems depends on the vehicle speed to avoid collisions but it is limited at a certain speed (40 $\mathrm{km} / \mathrm{h}$ ). Although these systems are intended to effectively reduce pedestrian injury outcomes, the collision avoidance performance of these systems remains limited.

The objective of this study is to understand the functionality of these active safety systems to prevent pedestrian crashes in terms of detecting the hazard and triggering Autonomous Emergency Braking (AEB). These issues explored in this paper are focused on the implication of using sensors with a wide field of view to detect pedestrians and the compromise between high effectiveness and a low false activation rate (C. G. Keller et al., 2011). This paper will address the problem of defining criteria for AEB activation in terms of:

- The trigger time for emergency maneuvers, and

- The remaining distance before impact.

Indeed, these two parameters encompass the most critical aspect of the performance and effects of pedestrian active safety systems because they affect the amount that the speed of the vehicle can be reduced prior to impact. In this study, the trigger times (relative to the time of impact) and the distance before impact at which a system is able to trigger are analyzed through the simulation of real world crash scenarios based on in-depth accident investigation.

\section{Materials and Methods}

In summary, the study uses a sample of crashes from in-depth accident databases. Each crash in the sample is reconstructed and modeled numerically. In parallel, a model representing the 
functioning of an AEB system has been established from a bibliographic research. Finally the system's model is coupled to the kinematic of the vehicle preceding the impact in order to evaluate the characteristics of the triggering of an AEB system in the sample of crashes.

\subsection{Accident data}

A sample of one hundred crashes was selected from two in-depth at-scene crash investigation databases: 60 cases were provided by CASR (Centre for Automotive Safety Research, University of Adelaide, Australia) and 40 cases were compiled from the IFSTTAR-LMA crash database (the laboratory of accident mechanism analysis of the French institute of science and technology for transport, development and networks, France). Both of these centres proceed in a similar way to perform in-depth investigations (Girard, 1993; Woolley et al., 2006). In particular, these in-depth studies consist of investigations by a multidisciplinary team, composed of a psychologist and a technician, and are made on the scene of the accident, at the same time as the intervention of the rescue services. Those performing the survey were asked to collect a maximum amount of data. Generally, this data consisted of the final positions of the vehicles, the marks left on the ground (tyres, fluids, debris, etc.), the point of impact on the vehicle (bonnet, windscreen, etc.), the direction of the impact, the weather conditions, the obstructions and also to collect statements of drivers, witnesses and make a record of any injuries on the basis of the medical report, etc. All this data was then pooled and compared in order to make an initial reconstruction of the accident and to make hypotheses regarding the process involved: direction of travel of the pedestrian, speed of the vehicle, etc. When the reconstruction proposed is in agreement with all indications available, it is adopted as being the most probable scenario. To summarize the data of the reconstruction, a global synthesis of the accident is drawn up by the investigators relating in details the story of the accident.

IFSTTAR-LMA crash cases include investigated accidents occurred between 1995 and 2011 near the townships of Salon-de-Provence and Aix-en-Provence. CASR accidents were investigated in the Adelaide Metropolitan Area in the period April 2002 to October 2005.

Data collected during each investigation included:

- Photographs and videos of the crash scene and vehicles involved;

- Statements of people involved in the crash, witnesses, and police;

- Details of the road environment, involved vehicles and pedestrians;

- Details of injuries from medical records;

- A site diagram of the accident drawing to scale including the marks observed on the scene (skid, debris, blood, etc.), the final position of the vehicle and the pedestrian, the estimated impact location and the estimated trajectories of the different subjects involved in the crash.

Some examples of these in-depth investigations have already been published (Hamdane et al., 2014).

The inclusion criteria of the cases used in this study were that the impact location on the roadway was known and the speed of the vehicle could be reliably assessed from standard crash reconstruction techniques. 
The accidents were systematically clustered into four distinct groups according to the predominant features of the pre-crash sequence, as they might relate to the performance of the sensing system. These groups were: obstacles which hid the pedestrian, curved vehicle trajectory, pedestrian crossing from the sidewalk and pedestrian crossing from the far side of the roadway.

\subsection{Accident modeling}

The first step in each was to graphically reconstruct it. The approximate trajectories of the vehicle and the pedestrian were extracted from the scaled accident diagram provided from the in-depth investigation. Obstacles that mask pedestrians were located also using the diagram. Then, a temporal reconstruction was set up to emulate the kinematics of both the vehicle and the pedestrian from a pre-defined initial state until the impact. The initial conditions of the crash-involved pedestrian and vehicle were defined. As the pedestrian walking speed is often missing in the in-depth accident databases, it was estimated from the speed of the $50^{\text {th }}$ percentile based on the age of the pedestrians (Huang et al., 2008; Montufar et al., 2007; Zhang et al., 2013) and considered as constant (Table 1). In particular, the speed change of the pedestrian when he/she starts walking has not been taken into account since this speed variation concerns less than the first half meter of his moving as shown by Zębala et al. (2012).

Table 1

Pedestrian speed according to the age (from [Huang et al, 2008])

\begin{tabular}{lll}
\hline \multirow{2}{*}{ Age } & \multicolumn{2}{l}{$50 \%$ speed $(\mathrm{m} / \mathrm{s})$} \\
\cline { 2 - 3 } & Walking & Running \\
\hline $5-9$ & 1.83 & 3.94 \\
\hline $10-14$ & 1.68 & 4.20 \\
\hline $15-19$ & 1.65 & 4.20 \\
\hline $20-29$ & 1.62 & 3.54 \\
\hline $30-39$ & 1.62 & 3.35 \\
\hline $40-44$ & 1.62 & 2.90 \\
\hline $45-49$ & 1.52 & 2.90 \\
\hline $50-54$ & 1.52 & 2.83 \\
\hline $55-59$ & 1.46 & 2.83 \\
\hline $60-64$ & 1.46 & 2.47 \\
\hline $65+$ & 1.28 & 2.47 \\
\hline
\end{tabular}

Similarly, the vehicle travel speed is also considered as constant over the chain of the precrash events in order to "rewind" and retrieve the position of the vehicle at the beginning of the accident scenario. This vehicle speed is given in the in-depth database and it is calculated or estimated using crash reconstruction methods (McLean et al., 1994; Serre et al., 2005). In cases where the driver did react by a hard braking, a constant braking deceleration was estimated for each accident case according to the road surface condition provided by the indepth database (for example, the deceleration is assumed to be $-8 \mathrm{~m} / \mathrm{s}^{2}$ for dry conditions and $-6 \mathrm{~m} / \mathrm{s}^{2}$ for bad conditions). Cases where the driver did brake gently were rejected from this study. Some other cases were added where the vehicle was stopped at an intersection before 
moving forward and colliding a pedestrian. In these cases, the acceleration of the vehicle was considered constant. When completed, the reconstruction includes the trajectories of both the vehicle and the pedestrian to locate in space and time the pedestrian relative to the vehicle for several seconds prior to the collision.

\subsection{Active safety system modeling}

Most of the systems found on the market use both a camera vision mounted beside the rear view mirror and radar located in the front end of the vehicle behind the grille (Coelingh et al., 2010; Hayashi et al., 2013; Meinecke et al., 2005; Scheunert et al., 2004). Data flows from the on-board sensors are merged during the vision processing. Sensors described as "time-offlight sensors" are designed to determine the distance between the vehicle and any obstacle. Imaging sensors are mainly used for detecting and tracking pedestrians (Gandhi and Trivedi, 2007). Pedestrians are accurately detected by the cameras when they are located less than $40 \mathrm{~m}$ from the vehicle (Meinecke et al., 2003). Hence, in the detection algorithm, it is the camera sensor which is the most relevant because it validates the pedestrian presence and the possibility of a collision. Therefore, in this study, it was decided that the detection system could be modeled solely by the camera placed behind the rear view mirror of the vehicle and without taking into account the effect of others sensors such as radar or scanner. It has been also assumed in this study that the camera is not affected by bad weather conditions. This model is also acceptable for stereo cameras located near the rear-vision mirror of the vehicle since these cameras analyze the forward scene by overlapping the view of both lenses.

Camera sensors have been modeled in this study by their range and Field Of View (FOV). Different FOVs were considered from $20^{\circ}$ to $45^{\circ}$ in order to evaluate its influence on detection. The range was fixed to $40 \mathrm{~m}$ according to the research conducted in the European project SAVE-U (Meinecke et al., 2003). The other characteristics of the camera like image processing or system lag are ignored since it is very difficult to get the required characteristics in the literature and consequently to model it numerically. Figure 1 illustrates a configuration of one camera as it has been considered in the accident modeling with a range and a FOV.

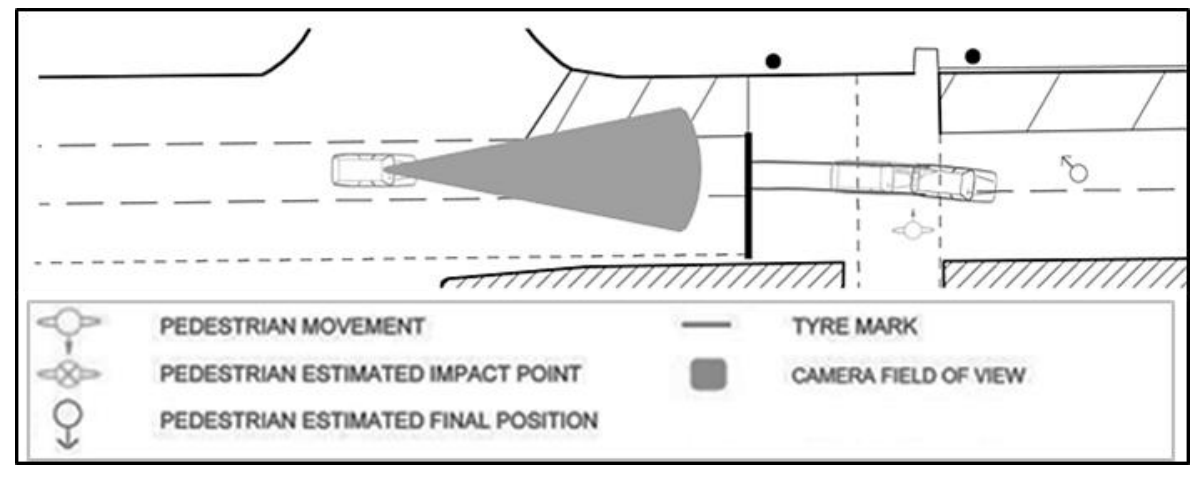

Figure 1: Scheme illustrating a crash representation including the active system

Concerning the actuators for triggering an emergency maneuver, they may include autonomous emergency braking and/or steering capabilities. But because the purpose of this paper is focusing on the detection of the pedestrian and the evaluation of the remaining distance before impact, the actuator considered was a generic AEB system. The generic AEB 
system was modeled with an appropriate level of average deceleration of $-8 \mathrm{~m} / \mathrm{s}^{2}$ (Brach and Brach, 2005; Byatt and Watts, 1981; Lechner and Ferrandez, 1990). This braking deceleration can be associated with "ideal" conditions: dry surface, good tires, efficient brake system and the like.

The brake force was assumed to have a step response excluding the existing system lag and the transient state. Figure 2 displays this brake model and compares it to a conventional one.

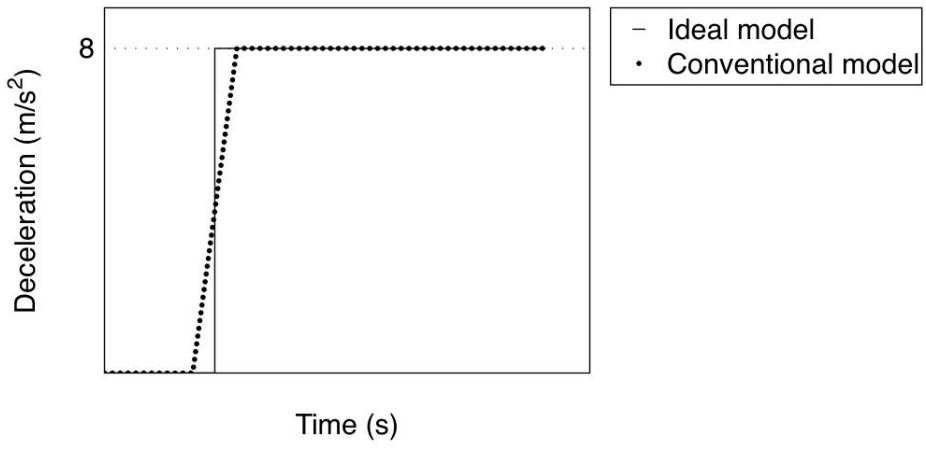

Figure 2: Comparison between the current and conventional brake model

\subsection{Simulation process}

Once the modeling had been set up, all the required components of an accident (crash environment, vehicle, pedestrian, and sensor) were simulated computationally. These components interacted in a customized virtual environment similar to the real world crash scenario (see Figure 1). The simulation emulated the trajectories of both the vehicle and the pedestrian starting from initial conditions until the crash using predefined time-steps. The program accounted for blind spots due to obstacles that mask the pedestrian and computed the instant when the pedestrian was detected by the sensor (that is to say located on the sensor's field of view but not hidden).

From the simulation, a set of data was extracted describing the characteristics of the accident at different pre-defined events. Data included the location of the pedestrian relative to the vehicle (the forward and lateral position), the speed of the vehicle (taking into account when the drivers accelerate or brake) and the Time-To-Collision (TTC). This last value is computed from the remaining travel distance before impact for the vehicle and its speed (Horst and Hogema, 1993).

In this paper, there is a focus on two specific events occurring in the crash sequence: the first instant when the pedestrian is visible by the sensor (considered as detection) and the last moment when the brakes need to be applied to avoid the crash.

For the detection, it is a question of determining the moment when the pedestrian is entirely inside the camera's Fields Of View and was not masked by any obstacle.

The ability of an AEB system to assist in complete collision avoidance is evaluated with reference to a time in the crash sequence that is the last time-to-brake (LTTB). It corresponds to the time when the vehicle is located at a distance $d_{\text {stop }}$ before the impact defined by the following equation: 


$$
d_{\text {stop }}=\frac{V^{2}}{2 *|a|}+d_{\text {offset }}
$$

where $V$ is the vehicle travelling speed $(\mathrm{m} / \mathrm{s}), a$ is the deceleration that fluctuates depending on the road conditions of the reconstructed accidents $\left(\mathrm{m} / \mathrm{s}^{2}\right), d_{\text {offset }}$ is the vehicle longitudinal clearance from pedestrian set at $0.3 \mathrm{~m}$.

At this sequence of the crash, an estimation of the avoidance rate of collisions is established for each camera FOV. It is calculated by verifying if the pedestrian is visible at LTTB even if the pedestrian is not yet on the roadway; i.e. it is possible for a system to avoid the crash (depending on the duration that the pedestrian is visible preceding the LTTB which is not measured here). Otherwise, if the pedestrian is out of the FOV or inside but masked by an obstacle, the case is considered mitigated (according to the moment of visibility before the collision) or unavoidable.

\section{Results}

3.1. Accident reconstruction results

Considering the environment of the crashes, specific factors are challenging the detection of the pedestrians. These factors are clustered into 4 scenarios.

- Scenario 1: pedestrian obscured by an obstacle (traffic, parked vehicle, tree, bin...);

- Scenario 2: pedestrian crossing through or after a turn;

- Scenario 3: pedestrian starting crossing from the sidewalk or nearby (from the right of the vehicle in France, from the left in Australia);

- Scenario 4: pedestrian crossing from the far side of the roadway (from the left of the vehicle in France, from the right in Australia).

Figure 3 illustrates each of these scenarios and gives their percentage distribution in the accident database.

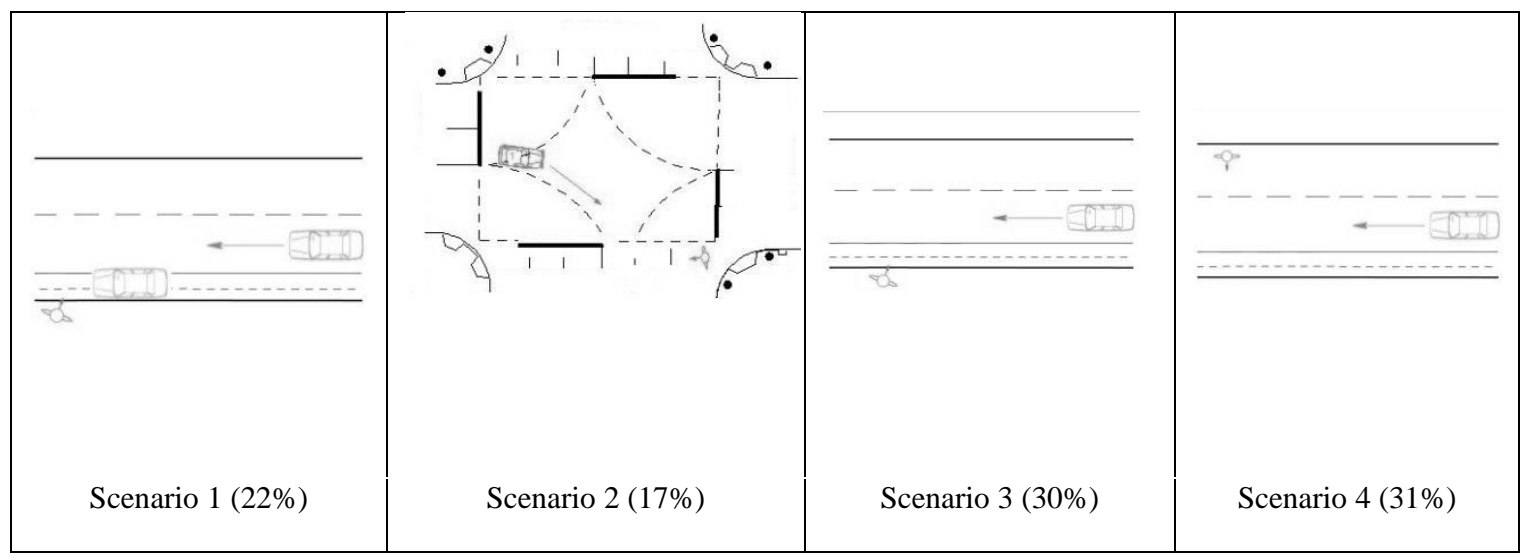

Figure 3: Description of the 4 crash scenarios

In order to better understand the relative trajectories between the vehicle and the pedestrian all along the accident scenario, the kinematic parameters were located in space and time. Figure 4 gives for the 100 accident reconstructions, the pedestrian position relatively to the vehicle position at different times which are respectively $2.5 \mathrm{~s}, 2 \mathrm{~s}, 1.5 \mathrm{~s}, 1 \mathrm{~s}$ and $0.5 \mathrm{~s}$ before the impact. This figure highlights in particular that until $1.5 \mathrm{~s}$, the positions of the pedestrians relatively to the vehicle are still scattered, and will largely not invoke any response from the AEB system. 
Then between $1.5 \mathrm{~s}$ and $0.5 \mathrm{~s}$, their positions are gathered mainly in front of the vehicle. This period between $1.5 \mathrm{~s}$ and $0.5 \mathrm{~s}$ TTC appears to be critical.

Moreover, these results show that the lateral position of the pedestrian a short time before collision appears to be very important because at $1 \mathrm{~s}$ before the impact, it can be observed that most of the pedestrians are located no more than $3 \mathrm{~m}$ of the side of the vehicle. Concerning their longitudinal position relative to the vehicle, the pedestrians are mainly to be found less than $20 \mathrm{~m}$ far from the front of the vehicle within $1 \mathrm{~s}$ TTC.
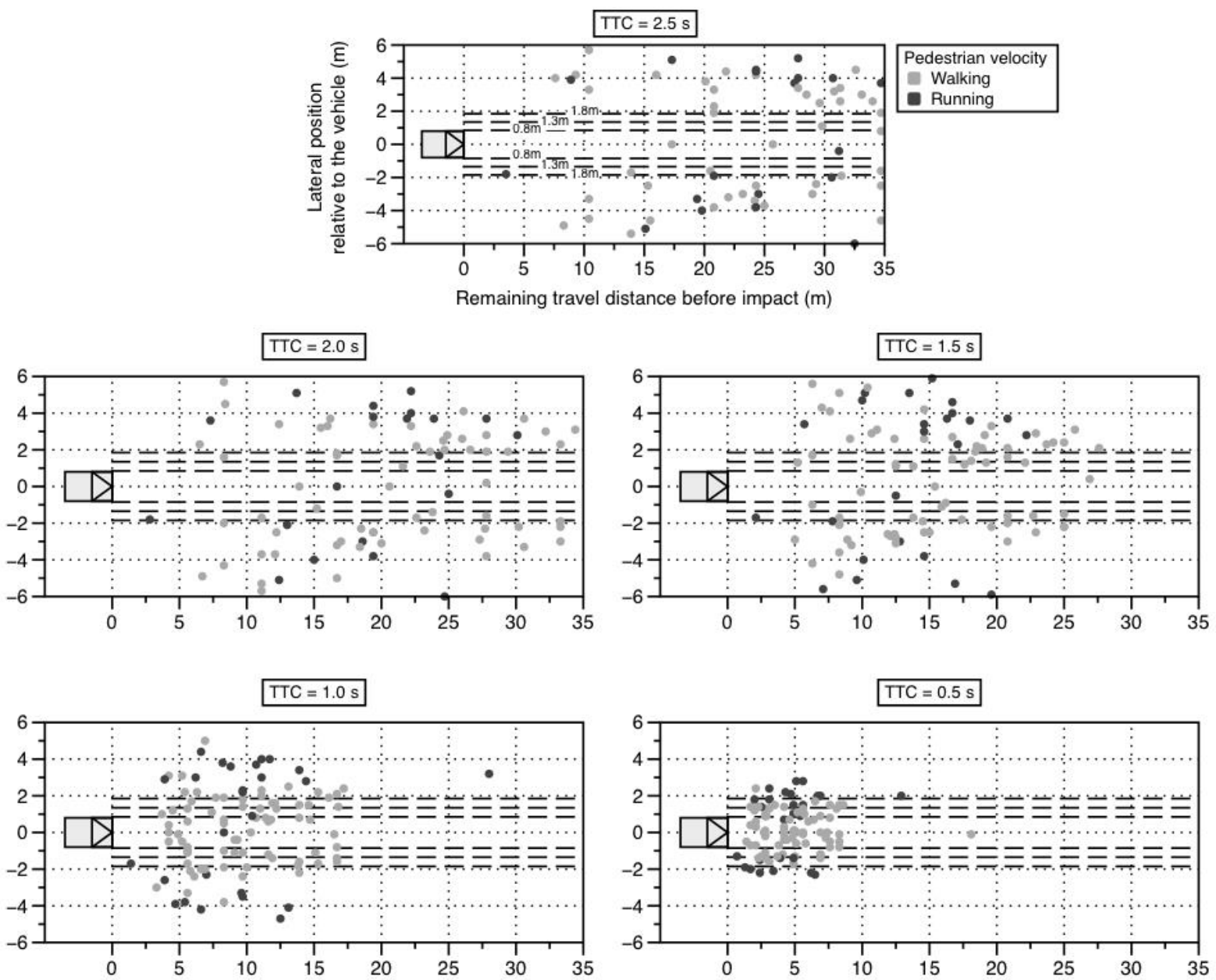

Figure 4: Pedestrian positions relatively to the vehicle at $2.5 \mathrm{~s}, 2 \mathrm{~s}, 1.5 \mathrm{~s}, 1 \mathrm{~s}$ and $0.5 \mathrm{~s}$ before impact

The travel speed of the vehicles ranged from 20 to $60 \mathrm{~km} / \mathrm{h}$ with an average value of $40 \mathrm{~km} / \mathrm{h}$ (S.D. $20.2 \mathrm{~km} / \mathrm{h}$ ). In $33 \%$ of cases, drivers perceive the hazard and then brake before the impact; the average impact speed was $32 \mathrm{~km} / \mathrm{h}$. More specifically, 23\% of drivers applied the brakes at a TTC greater than $1 \mathrm{~s}$ after perceiving the pedestrian located at a lateral distance greater than $0.5 \mathrm{~m}$ from the side of the vehicle.

The average walking speed of an adult is about $1.4 \mathrm{~m} / \mathrm{s}(\mathrm{SD}=0.39)$. Yet, there is a nonnegligible number of running pedestrians representing 24 cases; among that group, $62 \%$ are children which raise the running speed average to $3.5 \mathrm{~m} / \mathrm{s}(\mathrm{SD}=0.74)$. The specific scenario of these crashes is running children masked by another vehicle. Half of these running children 
are coming from the sidewalk. Three cases concern children hit at the beginning of their travel.

\subsection{Parameters relevant to pedestrian detection}

The detection of the pedestrians in each case is characterized by the Time-To-Collision (TTC) and the pedestrian location relative to the vehicle (the longitudinal and lateral position) at the first instant of detection. These detection parameters were evaluated for different Fields-OfView (FOV) of the sensor: $20^{\circ}, 25^{\circ}, 30^{\circ}, 35^{\circ}, 40^{\circ}$ and $45^{\circ}$.

Figure 5 gives the complementary cumulative frequencies for each of the three kinematic parameters according to the different FOV. From a general point of view, all the curves never reach $100 \%$ since there are about $10 \%$ of pedestrians that remain undetected till the crash. These undetected pedestrians are mainly due to a pedestrian location outside the sight of view of the sensor. These cases are collisions on the side of the vehicle.
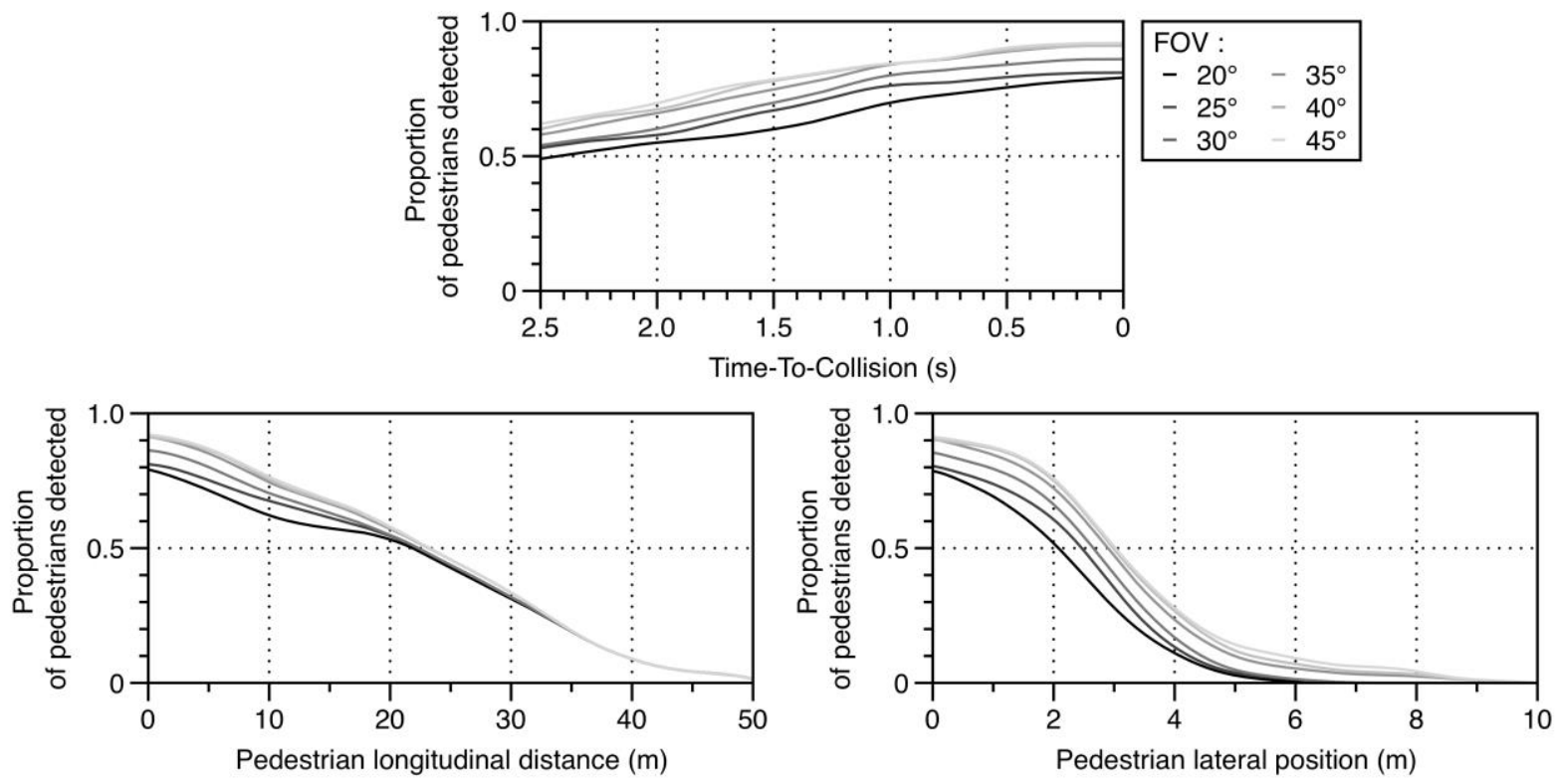

Figure 5: Rate of visible pedestrians for each kinematic parameter according to different FOVs

Concerning the time remaining before the impact, about $60 \%$ of pedestrians are visible by sensors with a FOV over $35^{\circ}$ at $2.5 \mathrm{~s}$ before impact (first time of the simulation). Because the number of visible pedestrian remains quite similar with upper FOV, it appears that the optimum FOV for the camera is above $35^{\circ}$. As important as it is, this optimized FOV is expected to detect beyond $80 \%$ of hazards 1 s before impact.

About the remaining distance before collision, it can be highlight that for all FOV more than half of the pedestrians are visible $20 \mathrm{~m}$ before the impact and so can be detected. The variation of the FOV has a main role only during the last $20 \mathrm{~m}$ with a better visibility according to a wider FOV. An angle of $35^{\circ}$ seems to be optimal again. It appears also that $10 \%$ of the pedestrians are never visible even with a FOV upper than $35^{\circ} .40 \mathrm{~m}$ before the impact, only $10 \%$ of pedestrians are visible. 
Considering the lateral distance, about $50 \%$ of pedestrians are visible with a FOV of $20^{\circ}$ from a distance in lateral of $2 \mathrm{~m}$. With a FOV upper than $35^{\circ}$, this rate is reached when pedestrians are located above $3 \mathrm{~m}$. For a FOV below of $35^{\circ}$, approximately no pedestrians are visible from a lateral distance of $6 \mathrm{~m}$. If it is considered a pedestrian situated on the far side of the road (i.e. at about $4.5 \mathrm{~m}$ in lateral), a FOV lower than $30^{\circ}$ allows a visible rate of $10 \%$ while for upper FOV, this rate reaches $20 \%$. This rate takes into account all visible pedestrians even if they are not yet on the roadway.

\subsection{Parameters relevant to AEB performance}

The kinematic parameters (TTC, longitudinal and lateral position of the pedestrian) are analyzed at the LTTB. This defines the requirements of an AEB system that can avoid the collision. The AEB performance refers to the crash avoidance rate obtained from the analysis of the 100 reconstructed crashes.

Figure 6 gives the cumulative frequency of the crash avoidance rate as a function of the kinematic parameters according to different sensor's FOV. It appears that $50 \%$ of accidents could be avoided if systems are able to be triggered 1s before the impact with a FOV upper than $35^{\circ}$. With a $20^{\circ} \mathrm{FOV}$, the avoidance rate decreases to $40 \%$. A threshold is reached at a LTTB equal to about $1.5 \mathrm{~s}$; beyond this LTTB value, there is little improvement in the rate at which crashes can be avoided. Most challenges concerning crash avoidance occur between $0.5 \mathrm{~s}$ and $1.5 \mathrm{~s}$ (corresponding to the sharp slope of the curve). With a FOV of $35^{\circ}$ or more, this threshold reaches an avoidance rate of $80 \%$. Once more, it seems that this FOV is an optimal value as it includes pedestrians not yet on the roadway.

Regarding the longitudinal distance, if the system could be triggered more than $20 \mathrm{~m}$ before the impact, about $60 \%$ of accidents in this sample would have been avoided with a FOV of $20^{\circ}$ (or $75 \%$ with a FOV of $35^{\circ}$ or more). Beyond $20 \mathrm{~m}$ there seems to be no more gain in the rate of avoidance.

For the lateral distance, similar patterns are observed except that the threshold corresponds to a distance of approximately $3 \mathrm{~m}$. The avoidance rate is affected by the FOV only at a distance beyond $2 \mathrm{~m}$. At this lateral distance, about $50 \%$ of accidents can be avoided. 

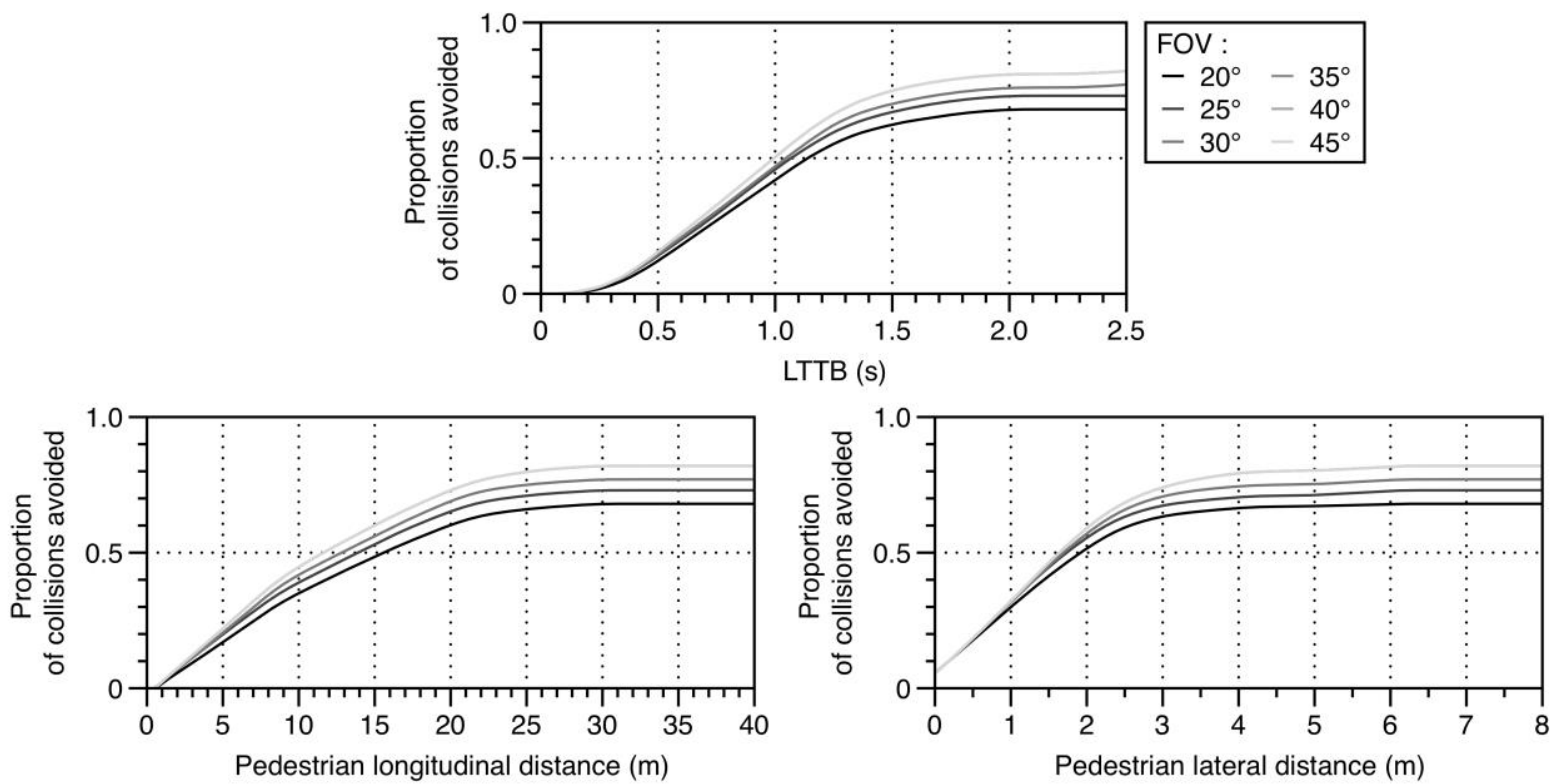

Figure 6: Pedestrian avoidance rate in function of kinematic parameters according to the camera FOV and the vehicle braking

In order to assess the available time for the system to react, the elapsed time between the instant when the pedestrian is visible $\left(\mathrm{t}_{\text {visible }}\right)$ and the LTTB was evaluated. Indeed this elapsed time corresponds to the duration available to detect the pedestrian and to trigger an AEB. This duration has been defined by Keller et al. (2011a) as the TTB (Time To Brake). Figure 7 shows the evolution of the complementary cumulative frequencies of avoided accidents according to this elapsed time and for the different FOV.

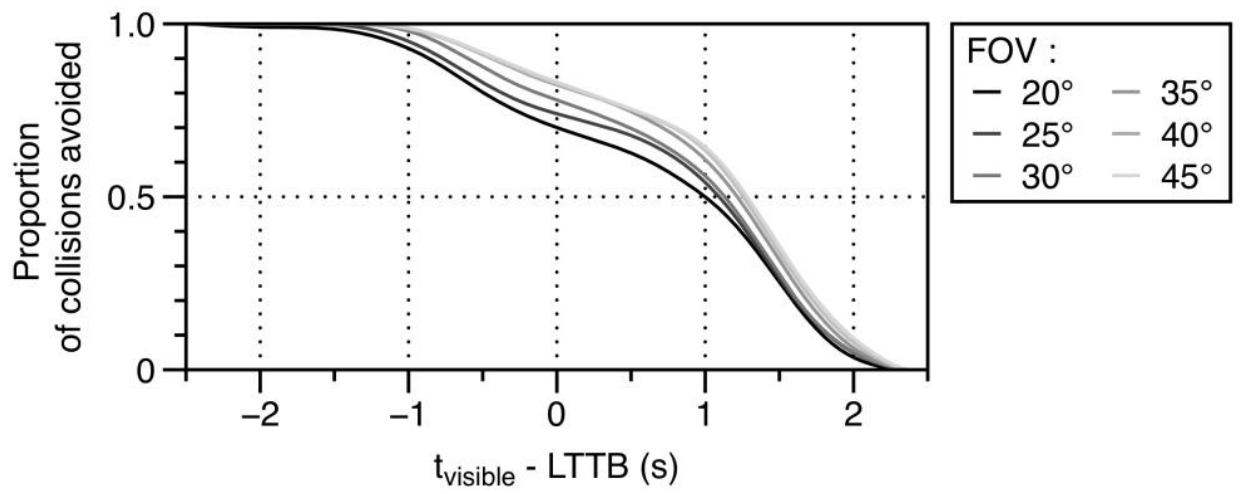

Figure 7: Complementary cumulative frequencies of the avoided accidents in function of the elapsed time from the visibility of the pedestrian to the LTTB for different FOV

These curves highlight that if the system is ideal, i.e. needs 0 second to react, the avoidance rate is comprised between $70 \%$ with a FOV of $20^{\circ}$ and $83 \%$ with a FOV of $35^{\circ}$.

With a FOV of $35^{\circ}$ or more, if the system requires $0.5 \mathrm{~s}$ for triggering (like the systems described by (Edwards et al., 2014), the avoidance rate decreases to $75 \%$. If the reaction time is $1 \mathrm{~s}$ (respectively $2 \mathrm{~s}$ ) the avoidance rate is $64 \%$ (respectively $~ 8 \%$ ). It is then noted that effectiveness rapidly declines for reaction times above one second. 
For fields-of-view above $35^{\circ}$, it is confirmed that the benefits of a system does not improve with increasing fields of view.

\section{Discussion}

This research is based on the understanding of the functioning of pedestrian active safety systems through real world crash reconstructions. A sample of 100 accidents involving pedestrians and passenger cars was gathered in order to illustrate the diversity of world crash configurations. Indeed, the sample is not only covering frontal impact configurations but also including side impacts along the fender of the vehicle.

Modelling the crash scenario depends of the availability of crash data required for the reconstruction. There is inevitably some fuzzy and even missing data to complete the reconstruction of a crash. This matter led to establish assumptions and define a procedure for modelling the different components of an accident in order to realise reliable computational simulations of the crash scenarios. To remove any doubt concerning the robustness of using data from in-depth investigation, a sensitivity study has been made on the assessments of two critical physical parameters: the speed of both pedestrian and vehicle. Results show that a variation of $+/-10 \%$ of these speeds can be neglected on the accident reconstruction. For example, for an accident with a car speed of $11,12 \mathrm{~m} / \mathrm{s}(40 \mathrm{~km} / \mathrm{h})$ and a pedestrian walking speed of $1,4 \mathrm{~m} / \mathrm{s}$, a car speed variation of $+/-5 \mathrm{~km} / \mathrm{h}$ and a pedestrian walking speed variation of $+/-0,4 \mathrm{~m} / \mathrm{s}$ induce a position of the pedestrian at LLTB of $+/-0,4 \mathrm{~m}$ on his trajectory. Similar analysis was conducted on the variance of the car deceleration and results show again a marginal effect.

The modeling of the detection function of an active safety system and its implementation in the computational simulation of crash scenarios allows the relevant parameters in detecting pedestrians to be highlighted. It has to be noted that the effect of lighting/weather conditions was not considered in the simulation of the crash scenarios. This constraint concerns more specifically the algorithms of detection and the limits of the technology. Additionally, no sensitive analysis was conducted in order to assess the false positive rate. This issue is also considered to be related to the algorithms of detection and probably differs from one active safety system to another. To include an assessment of the false positives to the simulation process, it suggests that the analysis should be conducted for a determined system.

In the set of 100 real accidents selected for this research, it appears that not all the pedestrians are visible prior to the crash. The remaining set of not visible pedestrians (about 10\%) corresponds mostly with scenarios where vehicles are turning (scenario S2) and obviously cases where pedestrians are masked by obstacles (scenario S1). These scenarios end up with side and front corner impact configurations.

Different FOV were evaluated for the sensor model. The evaluation highlighted that the rate of visible pedestrians is increasing with a wider FOV for the camera. This rate is about $79 \%$ for a $20^{\circ}$ cone angle while it reaches $92 \%$ for a $45^{\circ}$ cone angle. Additionally, from a $35^{\circ}$ FOV, a threshold in the visibility rate is observed. For example, at $1 \mathrm{~s}$ before impact about $80 \%$ of pedestrians could be detected with this FOV. Beyond it, the pattern of the visibility rate is similar for any kinematic parameter: the Time-To-Collision, the longitudinal and lateral 
pedestrian position relative to the vehicle. These results are complementary and in accordance with those of Rosén et al. (2010) which show a slight reduction of the severely injured (as well as fatality) for camera sensors with a FOV from $40^{\circ}$ to $180^{\circ}$. Thus, it can be considered that a FOV of $35^{\circ}$ is relevant for pedestrian detection. An expansion of this work could be interesting to study the influence of a $35^{\circ} \mathrm{FOV}$ on the AEB for vehicle-to-vehicle collisions. Regarding the triggering issue of an active safety system, the braking maneuvers were considered in this research but not the steering maneuver because this last emergency procedure must be handled with caution. Indeed, it is restricted by a considerable number of factors such as the traffic situation and an available clearance enabling a safe evasive action. Furthermore, drivers' behavior and driving situation in a bend are additional constraints that can cancel the steering maneuver. Moreover, if a front-end collision can be avoided by steering, it can possibly lead to a side collision when the pedestrian keeps moving (Hayashi et al., 2012).

The deployment of an AEB system (Autonomous Emergency Braking system) was assessed by computing the LTTB (Last Time To Brake). The system lag and the building rate of a full braking (i.e. the transient phase of an AEB system) were not considered in this research because not enough information was available. However, this delay can be taking into account in the processing time (Figure 7). For example, adding the braking system delay of $0.5 \mathrm{~s}$ to a processing time of $0.5 \mathrm{~s}$ leads to increase the elapsed time from the visibility of the pedestrian to the LTTB to $1 \mathrm{~s}$. So, according to Figure 7 , for a FOV of $35^{\circ}$, it would decrease the avoidance rate from $75 \%$ to $63 \%$. This rate could drop off considerably if the sum of the processing time and the delay is above 1s. Future systems will have to reduce these aforementioned parameters to improve their effectiveness.

Once the LTTB is calculated, it is possible to determine the effect of the FOV of a camerabased system in terms of avoidance or mitigated cases. As for detection, the avoidance rate increase with the FOV until reaching a threshold at $35^{\circ}$. This FOV can avoid approximately $50 \%$ of crashes if the system trigger at $1 \mathrm{~s}$ before impact. This rate is a little overestimated compared to the literature. Lindman et al. (2010) presented in a Case Study based on accident data from GIDAS the potential effectiveness of an active safety system developed by Volvo Cars (the CWAB-PD). It was estimated that CWAB-PD autonomous braking could avoid about $30 \%$ of all pedestrian accidents.

Concerning the reaction time of an active safety system (from detection of the hazard to the decision making and deployment of the emergency maneuver), the elapsed time between the instant when the pedestrian is visible and the LTTB was studied. During this elapsed time, it could be expected to alert the driver by a warning system (visual and/or audible alarm). Since a driver needs in average 1s to react (Lee et al., 2002), only cases with an available time more than $1 \mathrm{~s}$ can be considered. Such cases represent $50 \%$ of our database. So, among this rate, it is possible to avoid some accidents by prompting a response from the driver.

Finally, the results of this research can be used to establish specifications for an active safety system. Indeed, according to the Figure 7, it can be observed that the objective of $75 \%$ of avoidance rate require $0,5 \mathrm{~s}$ of reaction time for a $35^{\circ} \mathrm{FOV}$. 


\section{Conclusion}

The purpose of this paper was to highlight issues and challenges in pedestrian active safety through reconstruction of 100 real accident cases involving a vehicle and a pedestrian. The reconstructed crashes provided information concerning the crash configurations, the travel and impact speeds of vehicles, the velocity of pedestrians and their relative position in time and space, etc. In particular, the time between 0.5 and $1 \mathrm{~s}$ appeared as critical for the pedestrian safety.

The functionality of AEB systems were analyzed according to the reconstructed crash cases. The analysis considered different attribute of a system: the detection, reaction and triggering of the brakes. According to these attribute, a system based on a camera with a FOV of $35^{\circ}$ appeared relevant in terms of efficiency: high rates in detection ( $90 \%)$ and avoidance $(\sim 75 \%)$. Depending on the direction taken in using the graphs established in this research, it is possible to evaluate the efficiency of active safety systems as well as to define their specifications.

Next work will concern the evaluation of active safety systems in terms of crash mitigation (i.e. their potential effects on casualty reduction). The decrease of the impact speed induced by these systems will be assessed in order to identify the potential benefit of these systems on casualty reduction. This analysis will be carried using risk curves derived by logistic regression of the accident data. A coupled approach joining active and passive safety systems can be added in the future to establish assessed risk curves.

\section{Acknowledgements}

The authors would like to thank the both in-depth investigation teams of CASR (Centre for Automotive Safety Research, the University of Adelaide, Australia) and IFSTTAR-LMA (the Laboratory of accident mechanism analysis of the French institute of science and technology for transport, development and networks, Salon-de-Provence, France) for their help and cooperation in the reconstruction of the accidents.

\section{References}

Ando, K., Tanaka, N., 2013. An evaluation protocol for collision avoidance and mitigation systems and its application to safety estimation, in: Proceedings of the 23rd International Technical Conference on the Enhanced Safety of Vehicles. Seoul, Republic of Korea.

Brach, R.M., Brach, R.M., 2005. Vehicle accident analysis and reconstruction methods. SAE International.

Broggi, A., Cerri, P., Ghidoni, S., Grisleri, P., Ho Gi Jung, 2009. A New Approach to Urban Pedestrian Detection for Automatic Braking. Intell. Transp. Syst. IEEE Trans. On 10, 594-605.

Byatt, R., Watts, R., 1981. Manual of road accident investigation. Pitman.

Coelingh, E., Eidehall, A., Bengtsson, M., 2010. Collision Warning with Full Auto Brake and Pedestrian Detection - a practical example of Automatic Emergency Braking. Intell. 
Transp. Syst. ITSC 2010 13th Int. IEEE Conf. On 155-160. doi:10.1109/ITSC.2010.5625077

Ebner, A., Helmer, T., Samaha, R.R., Scullion, P., 2011. Identifying and Analyzing Reference Scenarios for the Development and Evaluation of Active Safety: Application to Preventive Pedestrian Safety. Int. J. Intell. Transp. Syst. Res. 9, 128-138. doi:10.1007/s13177-011-0035-z

Eckert, A., Hohm, A., Lueke, S., 2013. An integrated ADAS solution for pedestrian collision avoidance, in: Proceedings of the 23rd International Conference on the Enhanced Safety of Vehicles. Seoul, Republic of Korea, pp. 13-0298.

Edwards, M., Nathanson, A., Wisch, M., 2014. Estimate of Potential Benefit for Europe of Fitting Autonomous Emergency Braking (AEB) Systems for Pedestrian Protection to Passenger Cars. Traffic Inj. Prev. 15, S173-S182. doi:10.1080/15389588.2014.931579

Gandhi, T., Trivedi, M.M., 2007. Pedestrian Protection Systems: Issues, Survey, and Challenges. Intell. Transp. Syst. IEEE 8, 413-430. doi:10.1109/TITS.2007.903444

Girard, Y., 1993. In-depth investigation of accidents: the experience of INRETS at Salon de Provence. Presented at the The 6th ICTCT congress, Salzburg, Austria.

Hamdane, H., Serre, T., Anderson, R., Yerpez, J., 2014. Accident simulation and reconstruction for enhancing pedestrian safety: issues and challenges. Presented at the ESAR 2014 - 6th International Conference: Expert Symposium on Accident Research, Hannover, Germany.

Hayashi, H., Inomata, R., Fujishiro, R., Ouchi, Y., Suzuki, K., Nanami, T., 2013. Development of pre-crash safety system with pedestrian collision avoidance assist, in: Proceedings of the 23rd International Conference on the Enhanced Safety of Vehicles. Seoul, Republic of Korea, pp. 13-0271.

Hayashi, R., Isogai, J., Raksincharoensak, P., Nagai, M., 2012. Autonomous collision avoidance system by combined control of steering and braking using geometrically optimised vehicular trajectory. Veh. Syst. Dyn. 50, 151-168. doi:10.1080/00423114.2012.672748

Horst, A.R.A.V. der, Hogema, J.H., 1993. Time-to-collision and collision avoidance systems. Presented at the The 6th ICTCT congress, Salzburg, Austria.

Huang, S., Yang, J., Eklund, F., 2008. Evaluation of remote pedestrian sensor system based on the analysis of car-pedestrian accident scenarios. Saf. Sci. 46, 1345-1355. doi:10.1016/j.ssci.2007.08.004

Keller, C.G., Dang, T., Fritz, H., Joos, A., Rabe, C., Gavrila, D.M., 2011. Active Pedestrian Safety by Automatic Braking and Evasive Steering. Intell. Transp. Syst. IEEE Trans. On 12, 1292-1304.

Keller, C.G., Hermes, C., Gavrila, D.M., 2011. Will the pedestrian cross? Probabilistic path prediction based on learned motion features, in: Pattern Recognition. Springer, pp. 386-395.

Lechner, D., Ferrandez, F., 1990. Analysis and reconstruction of accident sequences. Presented at the XXIII FISITA Congress, ATA, Torino, Italy, pp. 931-939. 
Lee, J.D., McGehee, D.V., Brown, T.L., Reyes, M.L., 2002. Collision warning timing, driver distraction, and driver response to imminent rear-end collisions in a high-fidelity driving simulator. Hum. Factors 44, 314-334.

Lenard, J., Badea-Romero, A., Danton, R., 2014. Typical pedestrian accident scenarios for the development of autonomous emergency braking test protocols. Accid. Anal. Prev. 73, 73-80. doi:10.1016/j.aap.2014.08.012

Lindman, M., Oedblom, A., Bergvall, E., Eidehall, A., Svanberg, B., Lukaszewicz, T., 2010. Benefit estimation model for pedestrian auto brake functionality. Presented at the 4th International Conference on Expert Symposium on Accident Research, Hanover, Germany.

Lindman, M., Tivesten, E., 2006. A Method for Estimating the Benefit of Autonomous Braking Systems Using Traffic Accident Data (SAE Technical Paper No. 2006-010473). SAE International, Warrendale, PA.

McLean, J., Anderson, R.W.G., Farmer, M.J.B., Lee, B.H., Brooks, C.G., 1994. Vehicle travel speeds and the incidence of fatal pedestrian collisions. Federal Office of Road Safety, Transport and Communiations.

Meinecke, M.-M., Obojski, M.A., Gavrila, D., Marc, E., Morris, R., Töns, M., Letellier, L., 2003. Deliverable D6: Strategies in Terms of Vulnerable Road User Protection (EUProject SAVE-U No. Deliverable D6).

Meinecke, M.M., Obojski, M.A., Töns, M., Dehesa, M., 2005. SAVE-U: First Experiences with a Pre-Crash System for Enhancing Pedestrian Safety. Presented at the Intelligent Transportation Systems and Services, Hanover, Germany.

Montufar, J., Arango, J., Porter, M., Nakagawa, S., 2007. Pedestrians’ Normal Walking Speed and Speed When Crossing a Street. Transp. Res. Rec. J. Transp. Res. Board 90-97.

OECD/ITF, 2013. Road Safety Annual Report 2013.

Rosén, E., Källhammer, J.-E., Eriksson, D., Nentwich, M., Fredriksson, R., Smith, K., 2010. Pedestrian injury mitigation by autonomous braking. Accid. Anal. Prev. 42, 19491957. doi:10.1016/j.aap.2010.05.018

Scheunert, U., Cramer, H., Fardi, B., Wanielik, G., 2004. Multi sensor based tracking of pedestrians: a survey of suitable movement models. Presented at the Intelligent Vehicles Symposium, 2004 IEEE, pp. 774- 778.

Schram, R., Williams, A., van Ratingen, M., 2013. Implementation of autonomous emergency braking (AEB), the next step in Euro NCAP's safety assessment, in: Proceedings of the 23rd International Conference on the Enhanced Safety of Vehicles. Seoul, Republic of Korea.

Seiniger, P., Bartels, O., Pastor, C., Wisch, M., 2013. An open simulation approach to identify chances and limitations for vulnerable road user (VRU) active safety. Traffic Inj. Prev. 14 Suppl, S2-12. doi:10.1080/15389588.2013.797574

Serre, T., Bohn, M., Llari, M., Cavallero, C., Perrin, F.C., 2005. Detailed investigation and reconstructions of real accidents involving vulnerable road users. Berichte BASt Unterreihe Fahrzeugtechnik.

WHO, 2013. WHO | Global status report on road safety. 
Wisch, M., Seiniger, P., Edwards, M., Schaller, T., Pla, M., Aparicio, A., Geronimi, S., Lubbe, N., 2013. European project AsPeCSS - interim result: Development of test scenarios based on identified accident scenarios, in: Proceedings of the 23rd International Conference on the Enhanced Safety of Vehicles. Seoul, Republic of Korea, pp. 13-0405.

Woolley, J., Kloeden, C., Lindsay, V., Ponte, G., McLean, A., 2006. The Adelaide metropolitan indepth crash investigation study 2002-2005, in: 2006 Australasian Road Safety Research, Policing and Education Conference. Gold Coast, Australia.

Zębala, J., Ciępka, P., RezA, A., 2012. Pedestrian acceleration and speeds. Probl. Forensic Sci. 91, 227-234.

Zhang, X., Chen, P., Nakamura, H., Asano, M., 2013. Modeling pedestrian walking speed at signalized crosswalks considering crosswalk length and signal timing, in: Proceeding of the 10th International Conference of the Eastern Asia Society for Transportation Studies. Taipei, Taiwan. 